\title{
Two-dimensional Graphitic Metal Carbides: A New Class of Atomically Thin Carbon-based Crystals
}

\author{
Kah-Meng Yam ${ }^{[a] \dagger}$, Yongjie Zhang ${ }^{[a][b] \dagger}$, Na Guo ${ }^{[a]}$, Hui Deng ${ }^{[b] *}$, and Chun Zhang ${ }^{[a] c c] *}$ \\ ${ }^{[a]}$ Department of Physics and Centre for Advanced 2D Materials, National University \\ of Singapore, 2 Science Drive 3, Singapore 117551, Singapore \\ ${ }^{[b]}$ Department of Mechanical and Energy Engineering, Southern University of Science \\ and Technology, Shenzhen, 518055, Guangdong, China \\ ${ }^{[c]}$ Department of Chemistry, National University of Singapore, 3 Science Drive 3, \\ Singapore 117543, Singapore \\ ${ }^{[+]}$These authors contributed equally to this work \\ ${ }^{[*}$ Corresponding authors. E-mail: phyzc@nus.edu.sg
}

\begin{abstract}
The recent prediction of several single-atom thin two-dimensional (2D) graphitic metal carbides (g-MCs) with honeycomb-in-honeycomb $(\mathrm{HIH})$ structures is the first instance of designing 2D metal-carbon-only crystals from porous carbon allotropes via lattice reconstruction and these 2D g-MCs represent a new class of 2D carbon-based crystals. Here by extensive firstprinciples calculations, we show that this class of carbon-based crystals can be expanded to include 33 different members in total. These materials exhibit a myriad of properties, which can be useful in many different applications such as catalysis, spintronics, thermoelectrics, optoelectronics, etc. We reveal the chemical bonding in 2D g-MCs in terms of natural bonding orbitals to shed light on the origin of their unique electronic properties. In addition, these crystals show interesting but puzzling magnetic properties. A unified model to understand the origin of the magnetic properties of $2 \mathrm{D} \mathrm{g}-\mathrm{MCs}$ is hence proposed.
\end{abstract}

Keywords: 2D metal carbides • Honeycomb-in-honeycomb $\bullet$ 2D carbon-based crystals • Density functional theory 


\section{Introduction}

The realm of two-dimensional (2D) carbonaceous materials is burgeoning, spearheaded by graphene since its inception in 2004. [1] The exotic properties accompanying the reduced dimensionality in graphene were quick to be recognized; graphene possesses desirable properties such as a high surfaceto-volume ratio (SVR), high electrical and thermal conductivities, high melting point, and exceptional chemical inertness. ${ }^{[2]}$ Thus, considerable effort is devoted to the discovery of many other 2D carbon-based crystals, especially to overcome the shortcomings in graphene in various applications. In electronics, the difficulty in introducing a sizeable band gap has hindered the use of graphene in the generation of high-performance integrated logic circuits. ${ }^{[3]}$ The chemical inertness of graphene, while being a desirable property as a catalyst support, also translates to almost no intrinsic chemical reactivity. Thus, graphene must be subjected to treatments to strengthen the interaction between graphene and the catalytic active species, ${ }^{[4]}$ or to induce any catalytic activity in graphene. ${ }^{[5]}$

To date, there are three main classes of 2D carbon-based crystalline materials, namely carbon allotropes ${ }^{[6]}$ carbon pnictides, including carbon nitrides

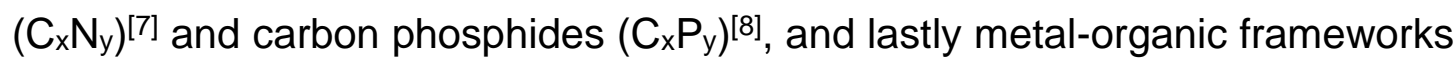
(MOFs) ${ }^{[0]}$. What is worth mentioning is that discovery and synthesis of materials involving direct bonding between metal and carbon seems to be challenging. This is from the common knowledge that the metal-carbon bonds are generally weak, in which the migration of atomically dispersed metal atoms in the lateral dimensions of the graphenic plane have been observed under the electron beam. ${ }^{[10]}$ As such, they are sporadically distributed on graphene, which will be unable to survive harsh conditions and easily aggregate when the concentration of metal atoms is too high. A notable exception is transition metal carbides (TMCs), belonging to another class of materials collectively known as MXenes[ ${ }^{[11]}$, in which the TMCs are intrinsically metallic unless upon surfacefunctionalization. ${ }^{[12]}$ Hence, 2D carbon-based crystals containing exposed metal atoms often involve anchor atoms to enhance the stability of the metal 
atoms in the network as in MOFs. ${ }^{\left[{ }^{[b]}\right]}$ Unfortunately, some MOFs also suffer from the relatively poor thermal stability, which would limit their practical applications. ${ }^{[13]}$

Such common knowledge mentioned earlier actuated the neoteric proposal of 12 highly stable $2 \mathrm{D}$ metal-carbon monolayers with $\mathrm{M}_{2} \mathrm{C}_{12}(\mathrm{M}=$ metal atom) being the chemical formula of the unit cell. ${ }^{[14]}$ These monolayers should be appropriately named as dimetal dodecarbide, or more succinctly, 2D graphitic metal carbides ( $g-M C s$ ) or $g-M C$ monolayers, which shall be the newest additions to the catalogue of $2 \mathrm{D}$ materials as the newest class of carbon-based crystals. Not only that the metal atoms directly bond to the carbon atoms and are periodically distributed in a graphenic lattice, but the metal atoms themselves form an interpenetrating honeycomb pattern with the honeycomb carbon lattice, which is referred to as the honeycomb-in-honeycomb $(\mathrm{HIH})$ structure.

Herein, an extensive computational screening involving non-radioactive metal elements from the $s-, p$ - and $d$-blocks of the Periodic Table were conducted for the possible species of $M$ that give stable monometallic $\mathrm{M}_{2} \mathrm{C}_{12}$ (two $\mathrm{M}$ atoms from the same element). The family of $2 \mathrm{D} g-\mathrm{MCs}$ vastly expanded to consist of 33 types, and the $d$-block members unambiguously exhibited a much richer palette of electronic and magnetic properties. The chemical bonding in these crystals was first attempted to be elucidated and found that while most crystals are predominantly covalent, a handful may also be stabilized via electrostatic interactions. Another highlight is that the intriguing magnetic properties exhibited by the materials can then be explained by a unified model that we proposed in this work. With the varying electronic band gaps and magnetic properties, these monolayers are expected to find possible applications in catalysis, spintronics, thermoelectrics, optoelectronics, etc. 


\section{Results and Discussion}

The first instance of designing 2D carbon-based crystals involving direct bonding between carbon and metal atoms (without the use of anchor atoms) from $2 \mathrm{D}$ porous carbon networks was demonstrated by $\mathrm{Li}$ et. al. ${ }^{[14]}$ The adsorption of $\mathrm{Fe}$ and some other metal atoms onto the triangular hollow sites of $\mathrm{Y}$-graphyne (GY) would distort the graphynic lattice to produce 2D g-MCs with a $\mathrm{HIH}$ structure: the $\mathrm{C}_{6}$ and $\mathrm{MC}_{5}$ moieties form the main honeycomb motifs resembling a graphenic lattice and the $M$ atoms alone also form larger interpenetrating honeycomb patterns. Furthermore, $\mathrm{HIH}$ structures comprise of structures that the metals reside on alternate plane of the carbon backbone, which would give a space group of $P-3$, those that the metal atoms are found on the same side (space group P6), and those that the carbon and metal atoms are on the same planar (space group $P 6 / m$ ), as shown in Figure 1 .

(a)

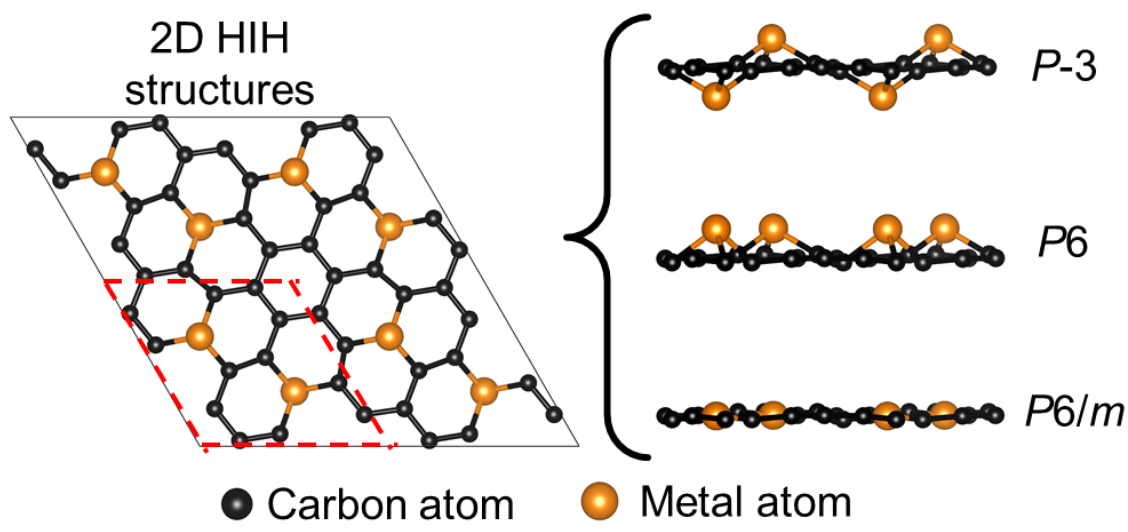

(b)

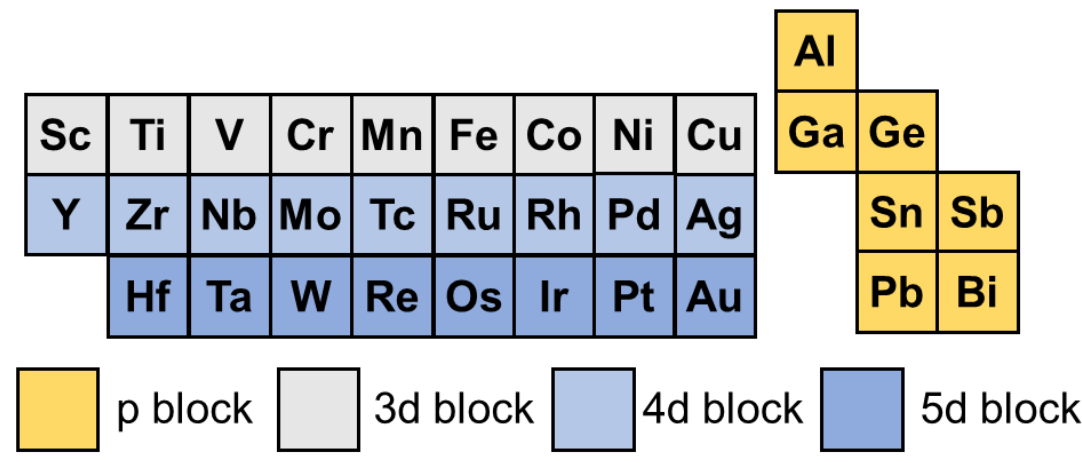

Figure 1. 2D g-MCs with $\mathrm{HIH}$ structures. (a) Top and side views of 2D-HIH structures, showing $P-3, P 6$, and $P 6 / m$ space groups, respectively. The unit cell of $2 \mathrm{D} g-\mathrm{MCs}$ is enclosed in red dash lines (2D-M $\mathrm{M}_{2} \mathrm{C}_{12}$ ). (b) Feasible metals for forming the stable $2 \mathrm{D}-\mathrm{HIH}$ structures. 
It is instructive to systematically investigate the possibility of the formation of such 2D g-MCs from the nonradioactive $s-, p$ - and $d$-block metal elements. In this work, we restrict to screening for a single type of metal species in $2 \mathrm{D} \mathrm{g}$ MCs as the scope of the search is already sufficiently wide, but we do not eliminate the possibility of bimetallic or other possible combinations of metal species in metal-carbon network, similarly to the very fact that bimetallic MXenes are also compounds synthesized. ${ }^{[15]}$ In the computational screening, we started with the $P-3$ structure of $\mathrm{Fe}_{2} \mathrm{C}_{12}$ monolayer in a hexagonal unit cell (i.e. $a=b, y=120^{\circ}$ ), and we replaced the Fe atoms with other metal atoms. The atoms and the cell parameters were optimized stepwise. The optimized $P 6$ and $P 6 / m$ structures were obtained in the similar fashion. Lastly, the ground state structures of the various $\mathrm{g}-\mathrm{MC}$ monolayers were ascertained by accepting the structure of the lowest energy. In addition, the energy of the ground state configuration was also checked against the optimized structure from adsorption of one metal atom on each hollow site in a unit cell of GY (with the unit cell referred to as $M_{2} / G Y$ ).

All feasible metals capable in forming stable 2D g-MCs with HIH structures are shown in Figure $1 \mathrm{~b}$ and listed in Table 1. Our results showed that $s$-block metal atoms are unable to form any stable $\mathrm{HIH}$ monolayers. Structurally, no $P 6 / m$ prototypes were found, for which the much larger atomic radii of the metal atoms than carbon atoms may be a very likely reason. ${ }^{[16]}$ Nevertheless, our results do not imply the preclusion of the existence of $P 6 / m$ structure: in the $2 D$ g-MCs subjected to strain, probably when grown on a suitable substrate with a significant lattice mismatch, or one may see in heterostructures that the metal atoms prefer to be planar with the carbon skeleton. On the other hand, $\mathrm{Ni}_{2} \mathrm{C}_{12}$ was the only monolayer found that assumes the $P 6$ space group structure but is almost degenerate with the $P-3$ isomer, and the rest were found to crystallize in the $P-3$ structure in the ground state. Additionally, if the $\mathrm{g}-\mathrm{MC}$ monolayers were to be grown on a substrate, then due to steric factors, the metal atoms would lie on the same side of the carbon skeleton. 
Table 1. Magnetic moments and band gaps of 2D g-MCs with HIH structures.

\begin{tabular}{|c|c|c|c|c|}
\hline & System ${ }^{[a]}$ & & $\begin{array}{c}\text { Magnetic } \\
\text { moment }\left(\mu_{\mathrm{B}}\right)\end{array}$ & $\begin{array}{c}\text { Band } \\
\text { gap (eV) }\end{array}$ \\
\hline \multirow{7}{*}{$\begin{array}{c}p \\
\text { block }\end{array}$} & $A l$ & $\mathrm{n}-\mathrm{SC}$ & - & 1.03 \\
\hline & $\mathrm{Ga}$ & $\mathrm{n}-\mathrm{SC}$ & - & 0.85 \\
\hline & $\mathrm{Ge}$ & $\mathrm{m}-\mathrm{M}$ & 0.42 & - \\
\hline & Sn & $n-M$ & - & - \\
\hline & $\mathrm{Sb}$ & $\mathrm{n}-\mathrm{SC}$ & - & 1.15 \\
\hline & $\mathrm{Pb}$ & $n-M$ & - & - \\
\hline & $\mathrm{Bi}$ & $\mathrm{n}-\mathrm{SC}$ & - & 1.30 \\
\hline \multirow{9}{*}{$\begin{array}{c}3 d \\
\text { block }\end{array}$} & Sc & $n-S C$ & - & 0.51 \\
\hline & $\mathrm{Ti}$ & $\mathrm{n}-\mathrm{SM}$ & - & 0 \\
\hline & $\mathrm{V}$ & $\mathrm{m}-\mathrm{M}$ & 2.44 & - \\
\hline & $\mathrm{Cr}$ & $\mathrm{m}-\mathrm{M}$ & 4.40 & - \\
\hline & $\mathrm{Mn}$ & $\mathrm{m}-\mathrm{HM}$ & 4.00 & $\uparrow: 0.29$ \\
\hline & $\mathrm{Fe}$ & $\mathrm{m}-\mathrm{HM}$ & 1.97 & $\uparrow: 0.30$ \\
\hline & Co & $\mathrm{n}-\mathrm{SC}$ & - & 0.19 \\
\hline & $\mathrm{Ni}$ & $n-M$ & - & - \\
\hline & $\mathrm{Cu}$ & $\mathrm{n}-\mathrm{SM}$ & - & 0 \\
\hline \multirow{9}{*}{$\begin{array}{c}4 d \\
\text { block }\end{array}$} & $\mathrm{Y}$ & $\mathrm{n}-\mathrm{SC}$ & - & 0.51 \\
\hline & $\mathrm{Zr}$ & $\mathrm{n}-\mathrm{SM}$ & - & 0 \\
\hline & $\mathrm{Nb}$ & $\mathrm{m}-\mathrm{M}$ & 2.00 & - \\
\hline & Mo & $\mathrm{n}-\mathrm{SC}$ & - & 0.16 \\
\hline & Tc & $\mathrm{m}-\mathrm{M}$ & 1.74 & - \\
\hline & $\mathrm{Ru}$ & $\mathrm{m}-\mathrm{M}$ & 0.62 & - \\
\hline & $\mathrm{Rh}$ & $\mathrm{n}-\mathrm{SC}$ & - & 0.42 \\
\hline & $\mathrm{Pd}$ & $\mathrm{m}-\mathrm{M}$ & 1.21 & - \\
\hline & $\mathrm{Ag}$ & $\mathrm{n}-\mathrm{SM}$ & - & 0 \\
\hline \multirow{8}{*}{$\begin{array}{c}5 d \\
\text { block }\end{array}$} & $\mathrm{Hf}$ & $\mathrm{n}-\mathrm{SM}$ & - & 0 \\
\hline & $\mathrm{Ta}$ & $\mathrm{m}-\mathrm{M}$ & 1.86 & - \\
\hline & W & $n-S C$ & - & 0.26 \\
\hline & $\operatorname{Re}$ & $\mathrm{m}-\mathrm{M}$ & 1.75 & - \\
\hline & Os & $\mathrm{m}-\mathrm{HM}$ & 2 & $\uparrow: 0.51$ \\
\hline & Ir & $\mathrm{n}-\mathrm{SC}$ & - & 0.47 \\
\hline & $\mathrm{Pt}$ & $\mathrm{m}-\mathrm{M}$ & 0.75 & - \\
\hline & $\mathrm{Au}$ & $\mathrm{n}-\mathrm{SM}$ & - & 0 \\
\hline
\end{tabular}

[a] The characteristics of various 2D g-MCs can be summarized as if they are magnetic $(m)$ or non-magnetic $(n)$ and if they are metallic $(M)$, half-metallic $(H M)$, semi-metallic $(S M)$, or semiconducting (SC). The magnetic moment per unit-cell is given for magnetic systems 
and likewise for the electronic band gap for semiconductors and semiconducting channels in $\mathrm{HM}$ crystals. For example, $2 \mathrm{D}-\mathrm{Fe}_{2} \mathrm{C}_{12}$ is a magnetic half-metal $(\mathrm{m}-\mathrm{HM})$ with a band gap of $0.30 \mathrm{eV}$ in the majority spin channel. Note that all the $\mathrm{M}_{2} \mathrm{C}_{12}$ unit cells have a space group of $P-3$ except for $2 \mathrm{D}-\mathrm{Ni}_{2} \mathrm{C}_{12}$. The arrow $\uparrow(\downarrow)$ denotes the majority (minority) spin channel for spin-polarized systems, where applicable.

The stability of these g-MC monolayers was preliminary judged based on two parameters, i.e., cohesive energy $\left(E_{c o h}\right)$ and reaction energy $\left(E_{r \times n}\right)$ per atom, where

$$
\begin{gathered}
E_{c o h}=\frac{E_{g-M C}-2 E_{M}-12 E_{C}}{14} \\
E_{r x n}=\frac{E_{g-M C}-E_{G Y}-2 E_{M}}{14}
\end{gathered}
$$

with $E_{g-M C}$ and $E_{G Y}$ denote the total energy of one unit cell of $\mathrm{g}-\mathrm{MC}$ monolayer and $G Y$, respectively, $E_{M}$ and $E_{C}$ represent the energy of an isolated metal atom and a single carbon atom in vacuum, respectively, and the denominator ' 14' being the total number of atoms in the unit cell. Negative values of $E_{c o h}$ indicate that it is more stable for the atoms to exist as the g-MC monolayers instead of individual atoms. On the other hand, $E_{r \times n}$ refers to the change in energy per atom when the monolayers form from $G Y$ upon adsorption of metal atoms:

$$
\mathrm{GY}+2 \mathrm{M} \rightarrow \mathrm{g}-\mathrm{MC}
$$

The negative values of $E_{\mathrm{rxn}}$ indicate that the synthetic processes of these $\mathrm{g}$ $M C$ monolayers are exothermic, in which the monolayer products are favored over the reactants. Only those metals atoms, as displayed in Figure 1b, that would give negative values of $E_{c o h}$ and $E_{r \times n}$ (given in Table S1) would be considered further. Those not shown are attributed to their inability to form the stable HIH g-MC monolayers, which we would not consider. We found that the calculated values of $E_{c o h}$ indicate that the stability of all $2 \mathrm{Dg}-\mathrm{MCs}$ is comparable to various common $2 \mathrm{D}$ materials, for example, $\mathrm{g}-\mathrm{C}_{3} \mathrm{~N}_{4}, \mathrm{MoS}$, and 
h-BN. ${ }^{[14]}$ In addition, it is also rational to conclude that the monolayers listed can be facilely synthesized from GY from the large negative values of $E_{\mathrm{rxn}}$. Besides, the energetics and structural parameters for the stable 2D g-MCs are presented in Table S1 too.

Incidentally, it was found that during our screening procedure, not all metal species would induce lattice reconstruction of GY, i.e. $M_{2} / G Y$ is a metastable state with a higher energy than the ground state $\mathrm{HIH} \mathrm{g-MC} \mathrm{monolayers.} \mathrm{We}$ can then understand this phenomenon by the fact that the lattice reconstruction of the graphynic lattice is a competition between the formation of the $\mathrm{M}-\mathrm{C}$ bonds and the breaking of the acetylenic $\pi$ bonds. From earlier thermochemical calculations and experiments, the energy required to break the acetylenic $\pi$ bonds in a GY unit cell was extrapolated to be around $10 \mathrm{eV} .{ }^{[17]}$ We tested the adsorption energies $\left(E_{\text {ads }}\right)$ of a metal atom (plotted in Figure $S 1$ ) on each of the two single-vacancies (SVs) in a $\sqrt{7} \times \sqrt{7}$ supercell of graphene(R7-gr), resembling the carbon skeleton in a g-MC monolayer unit-cell but without the metal atoms. $E_{a d s}$ is defined as follows,

$$
E_{a d s}=E_{g-M C}-2 E_{M}-E_{R 7-g r}
$$

where $E_{g-M C}$ and $E_{\mathrm{R} 7-\mathrm{gr}}$ denote the total energy of the final adsorption structures and the R7-gr with two SVs, respectively. This $E_{a d s}$ calculated gives an indication of the strength of the M-C bonds. Together with the plot in Figure $\mathrm{S} 1 \mathrm{c}$, we can conclude that lattice reconstruction would be more likely to happen under $\mathrm{OK}$ if the exothermicity in the formation of $\mathrm{M}-\mathrm{C}$ bonds is significantly greater than $\sim 10 \mathrm{eV}$. In fact, we found that all those monolayers with $\left|E_{\text {ads }}\right|<$ $10 \mathrm{eV}(\mathrm{M}=\mathrm{Cr}, \mathrm{Mn}, \mathrm{Cu}, \mathrm{Pd}, \mathrm{Ag}$ and $\mathrm{Au})$ have the $\mathrm{M}_{2} / \mathrm{GY}$ metastable states found. Nevertheless, under realistic experimental conditions at finite temperatures, the barrier for the conversion of the metastable $M_{2} / G Y$, which can be viewed as the kinetic product in the synthetic process, to the more stable $\mathrm{g}-\mathrm{MC}$ thermodynamic product should be easily overcome if the reaction time and the temperature are adequate. We would further elaborate on this point, exemplified by the $\mathrm{Cr}$ member. 
To verify our conjecture, we carried out spin-polarized ab initio molecular dynamics (AIMD) simulation, with each $\mathrm{Cr}$ atom initially placed in a triangular hollow site of a $2 \times 2 \times 1 \mathrm{GY}$ supercell on the same plane with the carbon atoms at $350 \mathrm{~K}$, as shown in Figure 2. One can observe the distortion of the graphynic lattice after $0.075 \mathrm{ps}$ and the formation of the graphenic lattice was complete at $0.48 \mathrm{ps}$. Furthermore, the irreversibility of the lattice reconstruction is evident from the fact that the graphenic lattice was still well-maintained till the end of the simulation, which lasted for $12 \mathrm{ps}$. This shows that the $\mathrm{Cr}_{2} / \mathrm{GY}$ can already be thermally activated at ambient conditions to form the stable $2 \mathrm{D}-\mathrm{Cr}_{2} \mathrm{C}_{12}$ monolayer. As a guidance to the experimentalists on the synthesis of $2 \mathrm{D} \mathrm{g}-\mathrm{MCs}$, a key process would be the rupture of the strong acetylenic $\pi$ bonds in GY, which we showed in our case here that temperature effects would aid in the activation of the in-plane $\mathrm{C}-\mathrm{C}$ bond vibrations, and in turn help to weaken those bonds to pave way for the formation of $\mathrm{M}-\mathrm{C}$ bonds.

(a)

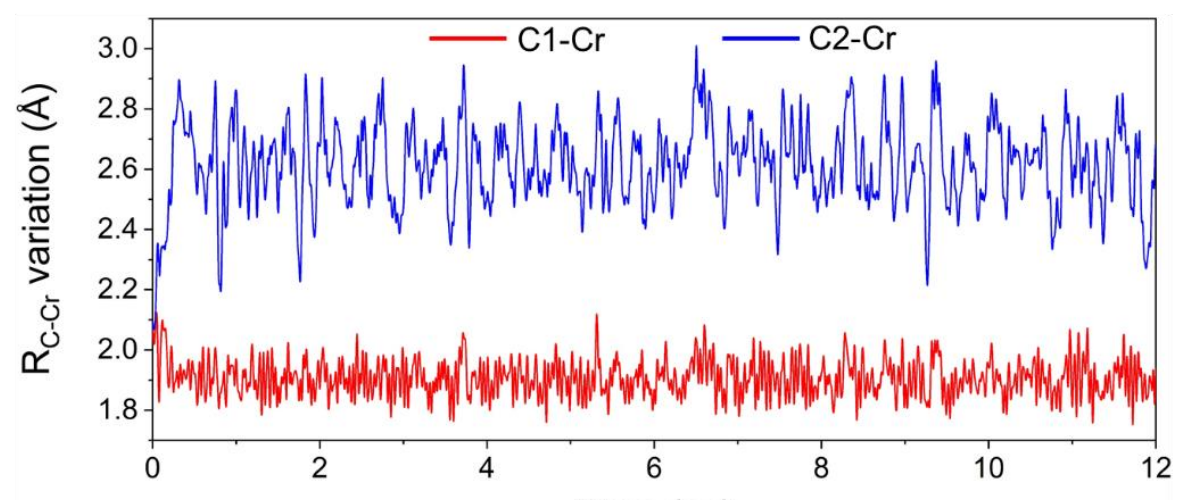

(b)

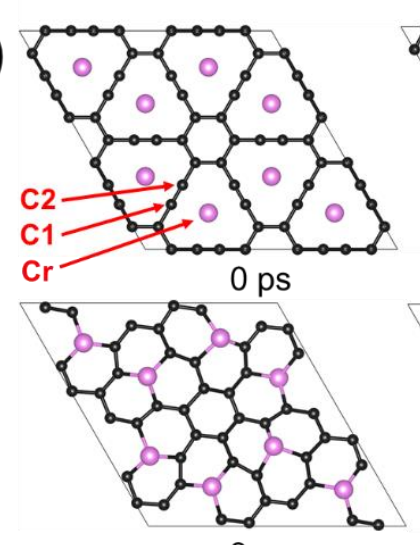

3 ps

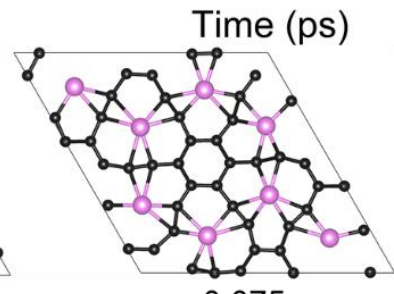

$$
0.075 \mathrm{ps}
$$

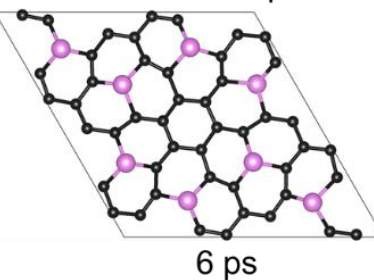

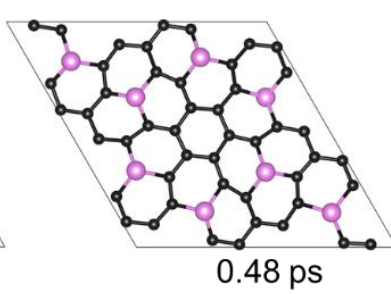

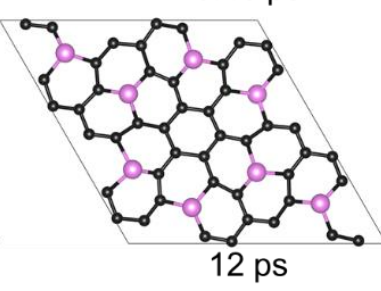

Figure 2. Spin-polarized ab initio molecular dynamics (AIMD) simulation results of the formation of $2 \mathrm{D}-\mathrm{Cr}_{2} \mathrm{C}_{12}$ monolayer. (a) $\mathrm{C} 1-\mathrm{Cr}$ and $\mathrm{C} 2-\mathrm{Cr}$ bond length variations at $350 \mathrm{~K}$. (b) Corresponding snapshots of the trajectories of the AIMD simulation at $350 \mathrm{~K}$. 
Subsequently, the electronic band structures of the stable 2D g-MCs listed in Table 1 were calculated and are all presented in Figure S2-S5 in the Supporting Information. Evidently, the crystals can be classified according to their magnetism, i.e., magnetic or non-magnetic, or in terms of their band gaps, i.e., metal, half-metal, semi-metal or semiconductor. Notably, no magnetic semiconductors were identified, and the half-metals are all magnetic materials. These crystals with different properties can undoubtedly have potential applications in different fields, which we would propose and highlight some of them herein. As demonstrated previously, the prototypical magnetic halfmetallic $\mathrm{Fe}_{2} \mathrm{C}_{12}$ can exhibit excellent catalytic activity towards $\mathrm{CO}$ oxidation reaction (COR), in which the reaction is expected to happen at ambient conditions. ${ }^{[14]}$ Hence, it would not surprising that other members of the 2D g$\mathrm{MCs}$, including nonmagnetic ones, can perform on par with or even outshine $\mathrm{Fe}_{2} \mathrm{C}_{12}$. Besides $\mathrm{COR}$, it is also expected that the magnetic members can catalyze nitrogen reduction reaction (NRR) and $\mathrm{CO}_{2}$ reduction reaction $\left(\mathrm{CO}_{2} \mathrm{RR}\right)$, in which local magnetic moments on the metal atoms play a crucial role in the activation of $\mathrm{N}_{2 .}{ }^{[18]}$ Furthermore, the uncommon half-metals are considered to have extremely broad application prospects in the field of $2 \mathrm{D}$ spintronics such as spin filtering and spin transistors. ${ }^{[19]}$ For the semiconducting ones, they can be used as thermoelectric materials, ${ }^{[20]}$ light-emitting diodes, ${ }^{[21]}$ and solar cells. $\left.{ }^{[8 a}, 21\right]$ The list is by no means exhaustive, and the applications of the materials await further detailed studies.

The $3 d$ and some other transition metal (TM) members of the g-MC monolayers exhibit a rich variety of electronic properties, which would be expected to be exploited as much as possible due to the low cost of the non-noble metals if they are realized experimentally. It would then be invaluable to dwell deep into the chemical bonding in this series of crystals, which would provide insights to the unique electronic properties of these $2 \mathrm{D} \mathrm{g}-\mathrm{MCs}$ and then the chemical design of functional materials for thermoelectric applications ${ }^{[22]}$ and molecular nanoelectronics. ${ }^{[23]}$ As such, the following sections would be devoted to analyzing the chemical bonding and origin of the magnetism in the $3 d \mathrm{TM}$ 
members of the 2D g-MCs with $P-3$ space group and we expect that these insights can then be easily extended to the other TM members.

To decipher the chemical bonding in the $3 d$ TM members $g-M C$ monolayers ( $M$ $=\mathrm{Sc}, \mathrm{Ti}, \mathrm{V}, \mathrm{Cr}, \mathrm{Mn}, \mathrm{Fe}, \mathrm{Co}$, and $\mathrm{Cu}$ ), we adopted the solid state adaptive natural density partitioning (SSAdNDP), which is an extension of the periodic natural bond orbital (PNBO) method. ${ }^{[24]}$ Compared to the PNBO method that can only be used to analyze chemical bonding in terms of lone pairs or one-center (1c) bonds, and two-center (2c) bonds, SSAdNDP can also interpret multi-center delocalized bonds. ${ }^{[25]}$ Such feature may then be capitalized to understand the metallicity in the various metals. ${ }^{[26]}$

As an illustration to the SSAdNDP searching process, we describe in detail the chemical bonding of the prototypical $\mathrm{Fe}_{2} \mathrm{C}_{12}$ and thus accordingly, chemical bonding in other 2D g-MCs can be interpreted in this way. The SSAdNDP results of the $3 d$ members of $\mathrm{g}-\mathrm{MC}$ monolayers are summarized in Table 2. One searches for the various NBOs by depleting all the valence electrons that are available for bonding in the simulation cell. For the magnetic $\mathrm{Fe}_{2} \mathrm{C}_{12}$ with each $\mathrm{C}$ atom and $\mathrm{Fe}$ atom contributing four and eight valence electrons respectively, and $2 \mu_{\mathrm{B}}$ per unit cell, one must assign a total of 33 majority spin electrons and 31 minority spin electrons. In the preliminary search, $21 \sigma$ bonds (including $\mathrm{C}-\mathrm{C}$ and $\mathrm{C}-\mathrm{Fe} \sigma$ bonds) were found for both spin channels, as shown in Figure 3a. In addition, six 1c and three $\mathrm{C}-\mathrm{C} \pi$ bonds were also found in the majority spin channel as shown in Figures $3 \mathrm{~b}$ and $3 \mathrm{c}$, respectively. On the other hand, there were no lone pair electrons found in the minority spin channel. That left us with three and seven electrons in majority and minority spin channels, respectively. In the custom search for multi-center bonds, three electrons from each spin channel were found in three six-center $(6 c) \pi$ bonds, involved in the localized $\pi$ electron cloud of the six-membered carbon ring, which survived and remained intact during the lattice reconstruction (Figure 3d). All the majority spin electrons were accounted for until this point. In the minority spin channel, six four-center (4c) bonds involving each Fe atom and the three surrounding $C$ 
atoms were found. As depicted in Figure $3 e$, these $4 \mathrm{c}$ bonds were formed between the $d$-orbitals on $\mathrm{Fe}$ atom and the $p$-orbitals from the $\mathrm{C}$ atoms. The remaining electron was then attributed to an extended eight-center (8c) bond (Figure 3f).

Table 2. Summary of the chemical bonding information in 2D g-MCs with HIH structures ( $\mathrm{M}=\mathrm{Sc}, \mathrm{Ti}, \mathrm{V}, \mathrm{Cr}, \mathrm{Mn}, \mathrm{Fe}, \mathrm{Co}$, and $\mathrm{Cu}$ ). The arrow $\uparrow(\downarrow)$ denotes the majority (minority) spin channel for spin-polarized systems.

\begin{tabular}{|c|c|c|c|c|c|c|c|c|}
\hline \multirow{2}{*}{\multicolumn{2}{|c|}{ System }} & \multirow{3}{*}{ Bonds } & \multicolumn{6}{|c|}{ Bond Types } \\
\hline & & & \multirow[t]{2}{*}{$1 \mathrm{c}$} & \multicolumn{2}{|r|}{$2 c$} & \multirow[t]{2}{*}{$4 c$} & \multirow[t]{2}{*}{$6 c$} & \multirow[t]{2}{*}{$8 c$} \\
\hline & & & & $\sigma$ & $\mathrm{C}-\mathrm{C} \pi$ & & & \\
\hline \multirow{2}{*}{ 흐 0} & Sc & 27 & 6 & 15 & 3 & - & 3 & - \\
\hline & $\mathrm{Cu}$ & 35 & 10 & 15 & 6 & - & 3 & 1 \\
\hline \multirow{10}{*}{$\begin{array}{l}\frac{\vec{\sigma}}{0} \\
\frac{0}{\sigma} \\
\partial \\
0\end{array}$} & $\mathrm{Ti}$ & 28 & - & 21 & 3 & - & 3 & 1 \\
\hline & \multirow{2}{*}{ V } & $\uparrow 30$ & - & 21 & - & 6 & 3 & - \\
\hline & & $\downarrow 28$ & - & 21 & 3 & - & 3 & 1 \\
\hline & \multirow{2}{*}{$\mathrm{Cr}$} & $\uparrow 32$ & 4 & 21 & - & 6 & - & 1 \\
\hline & & $\downarrow 28$ & - & 21 & 3 & - & 3 & 1 \\
\hline & \multirow{2}{*}{$\mathrm{Mn}$} & $\uparrow 33$ & 6 & 21 & 3 & - & 3 & - \\
\hline & & $\downarrow 29$ & - & 21 & 3 & - & 3 & 2 \\
\hline & \multirow{2}{*}{$\mathrm{Fe}$} & $\uparrow 33$ & 6 & 21 & 3 & - & 3 & - \\
\hline & & $\downarrow 31$ & - & 21 & - & 6 & 3 & 1 \\
\hline & Co & 33 & 6 & 21 & 3 & - & 3 & - \\
\hline
\end{tabular}


(a)

(c)
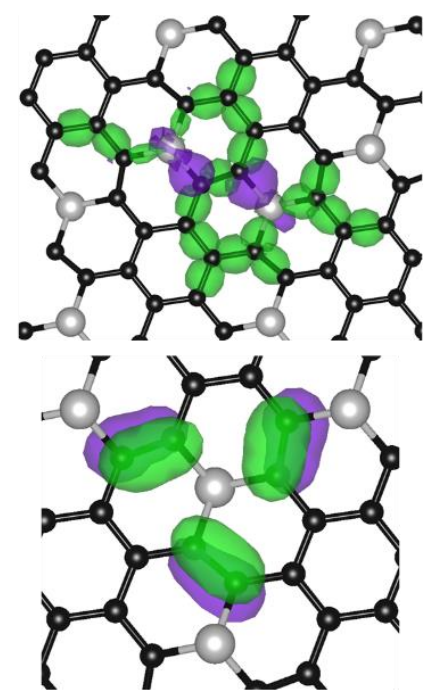

(f)

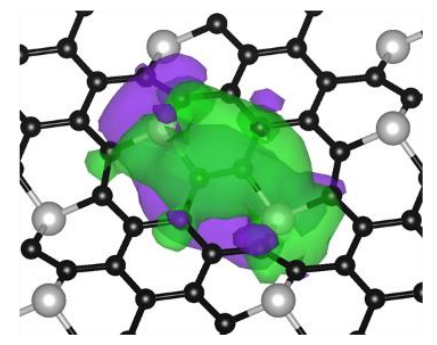

(b)

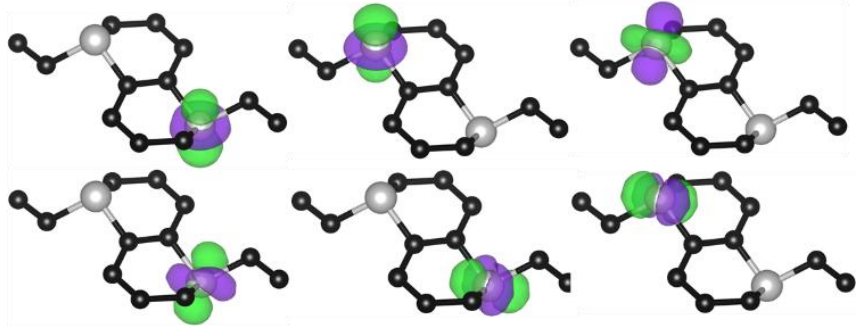

(d)
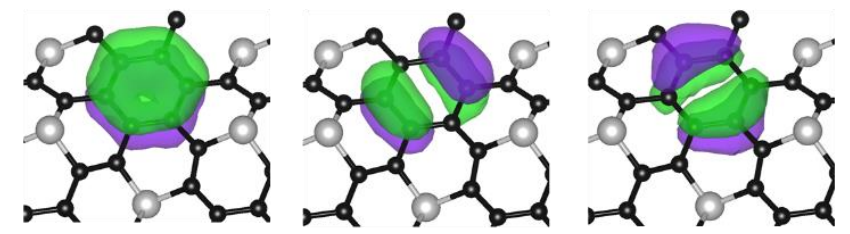

(e)

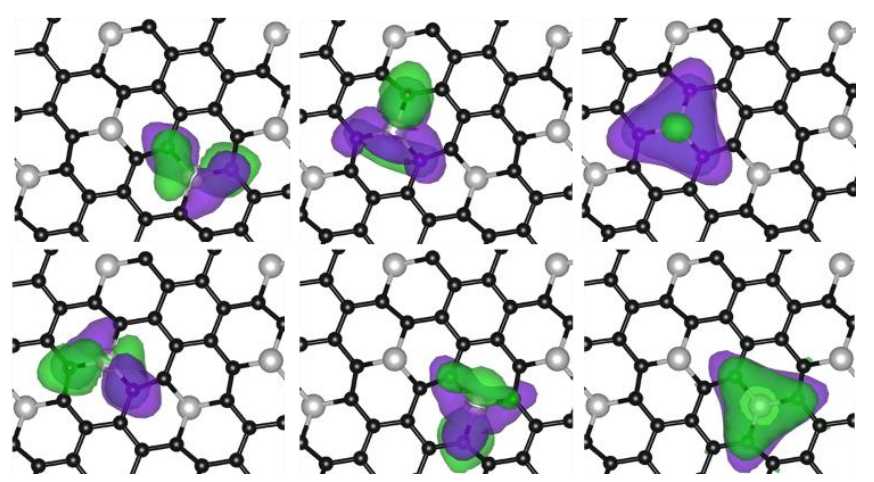

Figure 3. Bonding structures of $2 \mathrm{D}-\mathrm{Fe}_{2} \mathrm{C}_{12}$ monolayer. (a) $21 \sigma$ bonds ( $2 \mathrm{c}$ bonds), including $\mathrm{C}-\mathrm{C}$ and $\mathrm{C}-\mathrm{Fe} \sigma$ bonds, for the majority spin channel. The minority spin channel shares the same $\sigma$ bonds. (b) Six lone pair electrons (1c bonds) for the majority spin channel. (c) Three C-C $\pi$ bonds (2c bonds) for the majority spin channel. (d) Three $6 \mathrm{c}$ bonds for the majority spin channel. The minority spin channel shares the same $6 \mathrm{c}$ bonds. (e) Six C-M $\pi$ bonds (4c bonds) for the minority spin channel. (f) One $8 \mathrm{c}$ bond for the minority spin channel.

One can dichotomize the crystals to two distinct categories: ionic or covalent. In the first category, no M-C (covalent) bonds were found in the crystals, and the TM atoms interact with the carbon skeleton via predominantly electrostatic interactions by losing some charges to the carbon atoms. Amongst the $3 d$ members, $\mathrm{Sc}_{2} \mathrm{C}_{12}$ and $\mathrm{Cu}_{2} \mathrm{C}_{12}$ crystals belong to this category, whose electron localization function (ELF) were plotted in Figure S6. The value of ELF ranges between 0 and 1; an ELF value of a homogeneous electron gas is set to 0.5 and an ELF of 1 indicates that electrons are completely localized. It is clear from the 2D and three-dimensional (3D) ELF plots that there are no local maxima found between the metal and carbon atoms, indicating that there is no accumulation of electrons between the metal and carbon atoms in these 
systems. For $\mathrm{Sc}_{2} \mathrm{C}_{12}$, besides being able to locate the six lone pairs on the six $\mathrm{C}$ atoms surrounding the $\mathrm{Sc}$ ions, in which the additional charges on the $\mathrm{C}$ atoms were gained largely from the $\mathrm{Sc}$ atoms, the three $\mathrm{C}-\mathrm{C} \pi$ bonds, which constitute as the $\mathrm{C}-\mathrm{C}$ double bonds can also be identified as the 'banana bonds' in the ELF plot (Figure S6a). ${ }^{[27]}$ Similarly in the ELF plot of $\mathrm{Cu}_{2} \mathrm{C}_{12}$, the charges lost by $\mathrm{Cu}$ atoms to the $\mathrm{C}$ atoms can be seen as the small basins on those atoms and the $\mathrm{C}-\mathrm{C}$ triple bonds were identified as the 'banana bonds' (Figure $\mathrm{S} 6 \mathrm{e})$. However, the plot was drawn with a lower isosurface value of 0.57 such that the basins do not merge (cf. 0.80 for that of $\mathrm{Sc}_{2} \mathrm{C}_{12}$ ), indicating the higher delocalization of electrons in the metallic $\mathrm{Cu}-\mathrm{C}$ crystal, which also suggests the coexistence of ionic and metallic bonds ${ }^{[28]}$. The rest of the $3 d \mathrm{TM}$ members then fall under the category of covalent crystals, in which M-C $\sigma$ or $\pi$ bonds are found.

The NBOs found by SSAdNDP can be linked with the calculated band structures. Once again, we take the prototypical $2 \mathrm{D}-\mathrm{Fe}_{2} \mathrm{C}_{12}$ to demonstrate the relation between the NBOs found and its electronic band structure shown previously in Figure S3f. In the case of the semiconducting majority spin channel, all the bonds are well localized within the unit cell and cannot not couple with one another to form an extended electron cloud. This is unlike that of the metallic minority spin channel, in which the $4 \mathrm{c}$ bonds, which can couple with each other, and the $8 \mathrm{c}$ bond extend throughout the whole unit cell. These bonds underlie the metallicity of the system. In fact, for any of the metallic spin channels, there shall be either $4 \mathrm{c}$ or $8 \mathrm{c}$ bonds revealed by SSAdNDP. The features described for the Fe member are also shared by the other half-metallic $\mathrm{Mn}_{2} \mathrm{C}_{12}$, in which the semiconducting majority spin channel does not have any extended bonds, while two $8 \mathrm{c}$ bonds were found in the metallic minority spin channel (Figure S3e). By extension, we find that $\mathrm{Sc}_{2} \mathrm{C}_{12}$ and $\mathrm{Co}_{2} \mathrm{C}_{12}$ are semiconducting because their $\pi$ bonds are all localized, whereas $4 \mathrm{c}$ or $8 \mathrm{c}$ bonds can be found for $\mathrm{Ti}_{2} \mathrm{C}_{12}, \mathrm{~V}_{2} \mathrm{C}_{12}, \mathrm{Cr}_{2} \mathrm{C}_{12}$, and $\mathrm{Cu}_{2} \mathrm{C}_{12}$, as shown in Table 2 (cf. Figure $\mathrm{S} 3$ for the corresponding band structures). 
In addition to chemical bonding, the orbital origin of the magnetism in these g$\mathrm{MC}$ monolayers are also of particular interest. Our results showed that when the TM atoms in the covalent 2D g-MCs have incomplete $d$-subshell, then the material may be magnetic, and the spin densities are all largely localized on the metal atoms as shown in Figure S7. This indicates that a simplified local orbital picture describing the occupation of the $d$-orbitals contributed by two TM atoms, each surrounded by a single vacancy (SV) in a $\sqrt{7} \times \sqrt{7}$ graphene supercell, would suffice to elucidate the origin of the intrinsic magnetism. We shall describe the building of the model for the $P-3 \mathrm{HIH} \mathrm{g-MC} \mathrm{monolayers} \mathrm{in} \mathrm{detail}$ by considering ligand field splitting (TM-C), TM-TM, and exchange splitting (internal TM) interactions.

The model shall involve energy levels corresponding to a total of $10 d$ orbitals contributed by the two TM atoms per unit cell. Considering the TM-C interactions, each TM atom is in a $C_{3 v}$ point group and its five $d$-orbitals transform under the operations of the group to give a linear sum of the irreducible representations $\mathrm{A}+2 \mathrm{E}$. Out of the $d$-orbitals, $d_{z^{2}}$ has $\mathrm{A}$ symmetry, while the other four orbitals $d_{x z}, d_{y z}, d_{x y}$ and $d_{x^{2}-y^{2}}$ hybridize to form two doubly degenerate pairs E1 and E2 in a splitting mode of "E1-A-E2". Exemplified by $\mathrm{Fe}_{2} \mathrm{C}_{12}$ crystal, we identified 10 bands in each spin channel with significant contribution from the $d$-orbitals by calculating the projected density of states (PDOS) weighted band structure (Figure S8) and by plotting the banddecomposed wavefunctions at $\Gamma$ point in the first Brillouin zone (Figure S9). The crystal overlap Hamilton population (COHP) curve plotted for $\mathrm{Fe}_{2} \mathrm{C}_{12}$ (Figure S10) revealed that five which are lower in energy are bands resulting from bonding TM-C states while the rest are antibonding in nature. One can summarize these results as finding those $d$-bands at $\Gamma$ point follow a "E1-A-E2$E 2^{*}-A^{*}-E 1^{*}$ pattern in each spin channel.

While the two TM atoms are sufficiently far away to be unable to directly couple with each other, it seems that the two metal atoms in the unit cell correlated in the crystal because of the periodic boundary conditions. From the band- 
decomposed wavefunctions plotted and COHP calculations (Figures S9 and S10), the wavefunctions of the TM-C bonding states are centrosymmetric, while the wavefunctions of the antibonding states have odd symmetry. As depicted in the schematic in Figure S11, the carbon backbone functions as a bridge between the TM atoms to interact with one another, such that the wavefunctions of the $d$-orbitals can either overlap constructively to form pseudo-TM-TM bonding states or destructively to form the corresponding antibonding states.

By this point, we had already considered the TM-C and TM-TM interactions, which allows one to reduce the effects of the carbon backbone to two folds. Firstly, the backbone acts as a 'giant' ligand which provides the ligand field to split the otherwise degenerate $d$-orbitals. Secondly, it mediates the interactions between the two TM atoms in the unit cell. Our simplified local orbital picture would involve two TM atoms with the initially degenerate $10 d$ orbitals to split according to a "E1-A-E2-E2*-A*-E1"” pattern in each spin channel.

Another crucial interaction that should be included is the internal interactions in TM atom, which causes the exchange splitting between the two spin channels, and there are typically three different regimes. ${ }^{[29]}$ The first one is the weak interaction regime where the TM atom weakly interacts with the carbon scaffold. The exchange splitting is typically much larger than the ligand splitting, resulting in the largest magnetic moment obtained amongst the three regimes. The strong interaction regime is the second one, in which the exchange splitting is small compared to ligand splitting, resulting in orbitals of opposite spins to be filled first, and thus the system would be non-magnetic or weakly magnetic. The third regime describes an intermediate strength of the TM-C interaction, lying between that of the strong and the weak interaction regimes. We have the three sets of energy levels for the different cases as illustrated in Figure 4. The occupation of the energy levels was then worked out with the number of valence electrons contributed by the TM atoms to obtain the predicted magnetic moment in the unit cell. The occupation of the energy levels for each spin channel is presented in Table 3. One finds that regardless of the TM atoms, the 
predicted magnetic moment for the strong, intermediate, and weak cases would be 0,2 and $4 \mu_{\mathrm{B}}$ respectively. As the model cannot predict the TM-C interaction strength regime that the $\mathrm{g}-\mathrm{MC}$ monolayers fall under, prior knowledge of the calculated magnetic moment must be first obtained to qualitatively determine the TM-C interaction strength. Then by referring to Table 3 , the orbital origin of the magnetism can be inferred from the occupation of the energy levels. Note that the hypothetical $\mathrm{Ni}_{2} \mathrm{C}_{12}$ of $P-3$ space group was considered in this section for the completeness of the model. Also, for the group 10 ions, the model predicts the same magnetic moment of $2 \mu_{\mathrm{B}}$ for the intermediate and weak regimes as the majority spin channel would be fully filled.

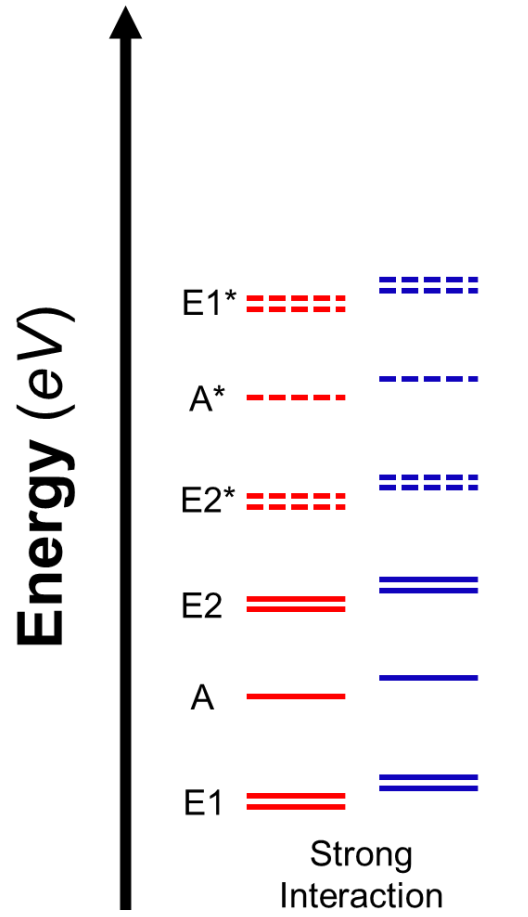

(a)

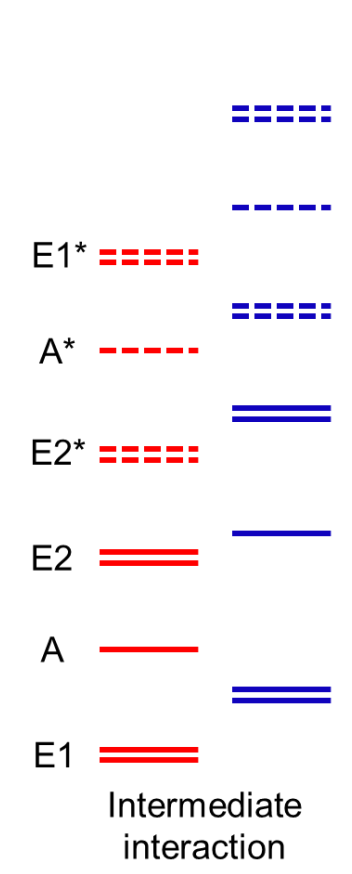

(b)

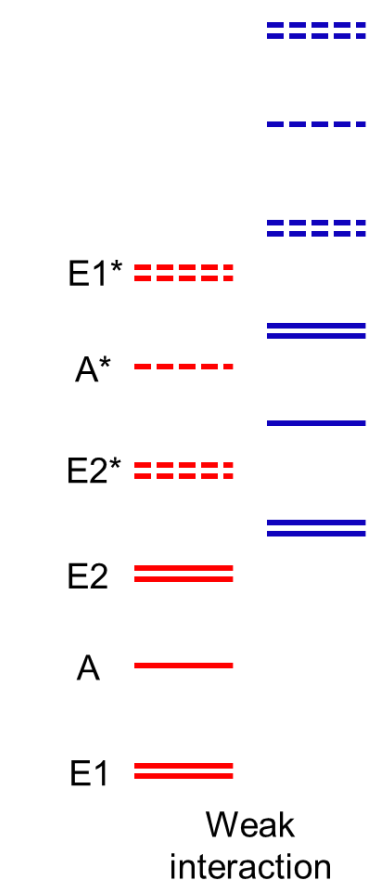

(c)

Figure 4. Schematic diagram of the energy levels for (a) strong interaction regime, (b) intermediate interaction regime, and (c) weak interaction regime. Red (blue) represents majority (minority) spin channel. Solid (dash) lines represent bonding (antibonding) state. 
Table 3. Magnetic moments of $2 \mathrm{D}$ g-MCs with $\mathrm{HIH}$ structures (P-3 space group) as a function of the electron configuration of the $\mathrm{M}+$ ions for strong, intermediate and weak TMC interaction regimes.

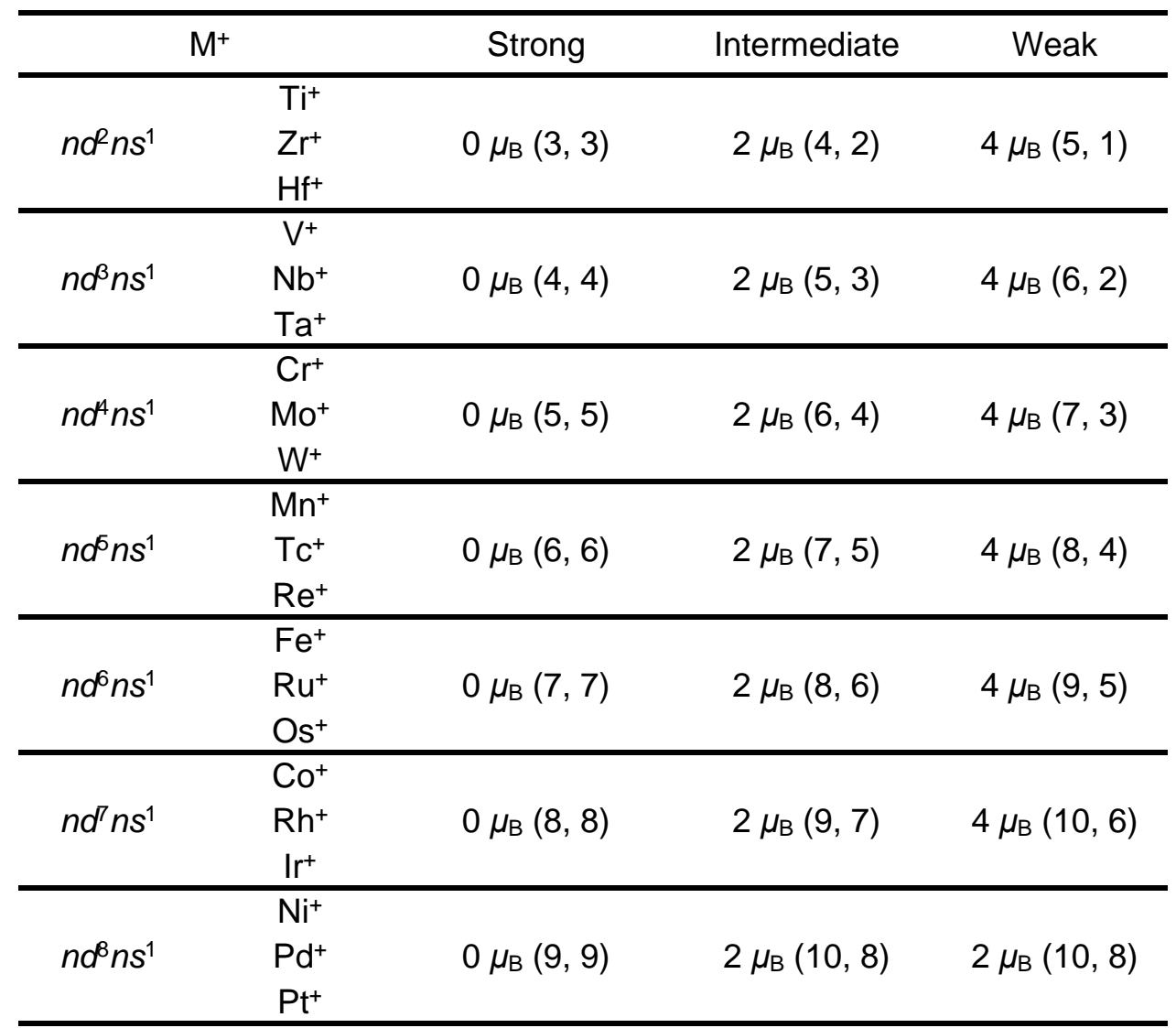

We then take the Group 8 members (Fe, Ru and Os) as illustration, with the electronic configuration of the atoms with +1 oxidation state in our crystals is $n d^{6} n s^{1}$. One would need to fill the 10 energy levels each from a spin channel according to the Aufbau principle with a total of 14 electrons. We filled the energy levels with 14 electrons for the three different regime models and found that the system would be spin-unpolarized in the strong interaction regime, and the intermediate and weak interaction regimes would predict a resulting magnetic moment of 2 and $4 \mu_{\mathrm{B}}$ respectively. Then, we find that $\mathrm{Fe}_{2} \mathrm{C}_{12}$ should belong to the case of intermediate interaction regime if one compares the predicted magnetic moment and the actual magnetic moment calculated, and $\mathrm{Os}_{2} \mathrm{C}_{12}$ with $2 \mu_{\mathrm{B}}$ per unit cell too. In contrast, $\mathrm{Ru}_{2} \mathrm{C}_{12}$ with a magnetic moment of only $0.62 \mu_{\mathrm{B}}$, which can be regarded as close to zero, should belong to the strong interaction regime. By comparing the occupation of the energy levels in 
the majority and minority spin channels, one can also deduce that the spin density in $\mathrm{Fe}_{2} \mathrm{C}_{12}$ and $\mathrm{Os}_{2} \mathrm{C}_{12}$ would roughly resemble that of hybrid of a predominant $A^{*}$ character and some $E 2^{*}$ characters as corroborated with the actual spin density plotted in Figure S7. Similarly, the spin density in $\mathrm{Ru}_{2} \mathrm{C}_{12}$ would consist of a mixture of $A^{*}$ and $E 2{ }^{*}$ characters. To confirm the correctness of our model, we compared the models with the corresponding electrons occupied for $\mathrm{Fe}_{2} \mathrm{C}_{12}, \mathrm{Ru}_{2} \mathrm{C}_{12}$ and $\mathrm{Os}_{2} \mathrm{C}_{12}$ (Figure $\mathrm{S} 12$ ) with the actual band structures calculated (Figures S3f, S4f, and S5e).

We find that $\mathrm{Fe}_{2} \mathrm{C}_{12}$ and $\mathrm{Os}_{2} \mathrm{C}_{12}$, both being magnetic half-metals, have very similar band structures. The majority spin channels in the two half-metals are semiconducting while the minority spin channels are metallic with the bands of E2* character being both partially filled and crossing the Fermi level $\left(E_{\mathrm{F}}\right)$ as the conduction bands. Comparing with the model, we note that the calculated $2 \mu_{\mathrm{B}}$ per unit cell means that out of the total of $14 d$-electrons mentioned earlier, eight would occupy the majority spin channel and all but the $E 1^{*}$ energy level would be occupied in the majority spin channel, up to the $A^{*}$ level. The remaining six would go to fill the minority spin channel, one of which would fill the doubly degenerate $\mathrm{E} 2{ }^{*}$ energy levels. This means that the $\mathrm{E} 2^{*}$ is only partially filled and hence this translates to the conduction bands as calculated.

In contrast, the actual band structure of $\mathrm{Ru}_{2} \mathrm{C}_{12}$ revealed that it is weakly magnetic although the model classifies the Ru-C interaction to be strong and predicts the material to be non-magnetic. While the model quantitatively cannot predict the extent of exchange splitting, it is nonetheless useful in understanding the orbital origin of the magnetism. The small magnetic moment would stem from the small exchange splitting in $\mathrm{Ru}_{2} \mathrm{C}_{12}$, which would cause the majority spin energy levels to be slightly lower than those in the minority spin channel. The $A^{*}$ level then manifests itself as a partially filled conduction band in the majority spin channel and the E2* level would be the partially filled conduction band in the minority spin channel. 
One can easily tell that not only can our model give magnetic moment close to the calculated one, but it can also provide insights to the orbital origin of the magnetism. Also, it can qualitatively describe the strength of the TM-C interactions as in the strong, intermediate, or weak regimes. This is achieved on the grounds that the formal charge of the TM ion is considered while determining the occupation of the energy levels, and that the order of the energy levels of the $d$ states in our models roughly parallels that of the occupied bands of interest at $\Gamma$ point in the actual band structures of the various $g-M C$ monolayer crystals. By comparing the actual magnetic moments calculated with that predicted by the model, we find that the $\mathrm{Ti}, \mathrm{Zr}, \mathrm{Hf}, \mathrm{Mo}, \mathrm{W}, \mathrm{Ru}, \mathrm{Co}, \mathrm{Rh}, \mathrm{Ir}$ and $\mathrm{Pt}$ members belong to the strong TM-C interaction regime; intermediate TM-C interaction members include V, Nb, Ta, Tc, Re, Fe, Os, Ni and Pd; and $\mathrm{Cr}$ and $\mathrm{Mn}$ members belong to the weak TM-C interaction regime. The model can be employed to understand other $3 d, 4 d$ and $5 d$ members of the covalent 2D g-MCs by undertaking the same procedure as described above.

\section{Conclusion}

Via first-principles calculation, we screened for all possible 2D monometallic gMCs with HIH structures from the $s$-, $p$ - and $d$-block metals with GY undergoing lattice reconstruction. While not all metal species would induce the lattice reconstruction at absolute zero, we suggested from our AIMD simulations that the conversion of the metastable state $M_{2} / G Y$ to the ground state $g-M C$ monolayer can be thermally activated. The crystals exhibit a plethora of properties: they can be dichotomized into intrinsically magnetic or nonmagnetic, or categorized into metals, half-metals, semi-metals, and semiconductors. We therefore employed the PNBO method and its extension SSAdNDP to reveal the underlying chemical bonding in these 2D g-MCs to give insights to the unique electronic properties, and for future design and understanding of functional materials in various applications such as catalysis, spintronics, thermoelectrics, optoelectronics, etc. It was also found that the metallicity in our systems is related to the delocalized $4 \mathrm{c}$ or $8 \mathrm{c}$ bonds. Additionally, we built a unified model to understand the orbital origin of the 
magnetic properties of $2 \mathrm{D} g-\mathrm{MCs}$. Not only can our model predict magnetic moments close to the calculated one and give insights to the orbital origin of the magnetism, but also qualitatively describe the strength of the TM-C interactions as in strong, intermediate, or weak regimes. These results provide a fundamental and systematic understanding of the bonding, electronic, and magnetic properties of $2 \mathrm{D}$ g-MCs, which may stimulate the design, synthesis, and application of such carbon-based crystals. On a broader note, owing to the unexpectedly strong metal-carbon interactions leading to the reconstruction of carbon lattice, we expect that more novel 2D metal-carbon-only crystals can also be realized from other porous carbon allotropes.

\section{Acknowledgements}

CZ acknowledges the support from Ministry of Education of Singapore (R-723000-029-112), NUS academic research fund (R-144-000-449-114) and NUS green energy program (R-143-000-A63-114). Computations were done with the NUS High Performance Computing (HPC) facilities.

\section{Conflict of Interests}

The authors declare no conflict of interests.

\section{References}

[1] K. S. Novoselov, D. Jiang, F. Schedin, T. J. Booth, V. V. Khotkevich, S. V. Morozov, A. K. Geim, Proc. Natl. Acad. Sci. U.S.A. 2005, 102, 10451-10453.

[2] K. Yam, N. Guo, Z. Jiang, S. Li, C. Zhang, Catalysts 2020, 10, 53.

[3] K. S. Novoselov, V. I. Fal'ko, L. Colombo, P. R. Gellert, M. G. Schwab, K. Kim, Nature 2012, 490, 192-200.

[4] a) Y.-H. Lu, M. Zhou, C. Zhang, Y.-P. Feng, J. Phys. Chem. C 2009, 113, 2015620160; b) M. Zhou, A. Zhang, Z. Dai, Y. P. Feng, C. Zhang, J. Phys. Chem. C 2010, $114,16541-16546$.

[5] a) N. Guo, K. M. Yam, C. Zhang, npj 2D Mater. Appl. 2018, 2, 1-6; b) K.-M. Yam, N. Guo, Z. Jiang, S. Li, C. Zhang, J. Phys. Chem. C 2020, 124, 18126-18131. 
[6] a) F. Diederich, M. Kivala, Adv. Mater. 2010, 22, 803-812; b) Q. Fan, L. Yan, M. W. T. O. Krejčí, S. Dimosthenous, S. R. Kachel, M. Chen, A. S. Foster, U. Koert, P. Liljeroth, J. M. Gottfried, Science 2021, 372, 852-856.

[7] a) T. S. Miller, A. B. Jorge, T. M. Suter, A. Sella, F. Corà, P. F. McMillan, Phys. Chem. Chem. Phys. 2017, 19, 15613-15638; b) D. Wang, Z. Wang, W. Liu, Arramel, J. Zhou, Y. P. Feng, K. P. Loh, J. Wu, A. T. S. Wee, ACS Nano 2020, 14, 14008-14016; c) L. Tan, C. Nie, Z. Ao, H. Sun, T. An, S. Wang, J. Mater. Chem. A 2021, 9, 17-33.

[8] a) T. Yu, Z. Zhao, Y. Sun, A. Bergara, J. Lin, S. Zhang, H. Xu, L. Zhang, G. Yang, Y. Liu, J. Am. Chem. Soc. 2019, 141, 1599-1605; b) X. Ma, J. Zhou, T. Yang, D. Li, Y. P. Feng, Phys. Rev. Mater. 2021, 5.

[9] a) R. J. Kuppler, D. J. Timmons, Q.-R. Fang, J.-R. Li, T. A. Makal, M. D. Young, D. Yuan, D. Zhao, W. Zhuang, H.-C. Zhou, Coord. Chem. Rev. 2009, 253, 3042-3066;

b) G. Zhu, Q. Sun, Comput. Mater. Sci. 2016, 112, 492-502.

[10] a) Y. Gan, L. Sun, F. Banhart, Small 2008, 4, 587-591; b) A. W. Robertson, B. Montanari, K. He, J. Kim, C. S. Allen, Y. A. Wu, J. Olivier, J. Neethling, N. Harrison, A. I. Kirkland, J. H. Warner, Nano Lett. 2013, 13, 1468-1475.

[11] Y. Gogotsi, B. Anasori, ACS Nano 2019, 13, 8491-8494.

[12] A. Champagne, J.-C. Charlier, J. Phys.: Mater. 2020, 3, 032006.

[13] C. Healy, K. M. Patil, B. H. Wilson, L. Hermanspahn, N. C. Harvey-Reid, B. I. Howard, C. Kleinjan, J. Kolien, F. Payet, S. G. Telfer, P. E. Kruger, T. D. Bennett, Coord. Chem. Rev. 2020, 419, 213388.

[14] S. Li, K.-M. Yam, N. Guo, Y. Zhao, C. Zhang, npj 2D Mater. Appl. 2021, 5, 1-7.

[15] M. Naguib, O. Mashtalir, J. Carle, V. Presser, J. Lu, L. Hultman, Y. Gogotsi, M. W. Barsoum, ACS Nano 2012, 6, 1322-1331.

[16] A. V. Krasheninnikov, P. O. Lehtinen, A. S. Foster, P. Pyykko, R. M. Nieminen, Phys. Rev. Lett. 2009, 102, 126807-126810.

[17] A. Nicolaides, W. T. Borden, J. Am. Chem. Soc. 1990, 113, 6750-6755.

[18] X. F. Li, Q. K. Li, J. Cheng, L. Liu, Q. Yan, Y. Wu, X. H. Zhang, Z. Y. Wang, Q. Qiu, Y. Luo, J. Am. Chem. Soc. 2016, 138, 8706-8709.

[19] a) B. Pradines, L. Calmels, R. Arras, Phys. Rev. Appl. 2021, 15, 034009; b) G. Bhattacharyya, P. Garg, P. Bhauriyal, B. Pathak, ACS Appl. Nano Mater. 2019, 2, 6152-6161.

[20] K. Kanahashi, J. Pu, T. Takenobu, Adv. Energy Mater. 2019, 10, 1902842.

[21] Q. Ma, G. Ren, K. Xu, J. Z. Ou, Adv. Opt. Mater. 2020, 9, 2001313.

[22] W. G. Zeier, A. Zevalkink, Z. M. Gibbs, G. Hautier, M. G. Kanatzidis, G. J. Snyder, Angew. Chem. Int. Ed. 2016, 55, 6826-6841.

[23] T. A. Su, M. Neupane, M. L. Steigerwald, L. Venkataraman, C. Nuckolls, Nat. Rev. Mater. 2016, 1, 1-15.

[24] a) E. D. Glendening, C. R. Landis, F. Weinhold, WIREs Comput. Mol. Sci. 2011, 2, 1-42; b) B. D. Dunnington, J. R. Schmidt, J. Chem. Theory Comput. 2012, 8, 19021911.

[25] T. R. Galeev, B. D. Dunnington, J. R. Schmidt, A. I. Boldyrev, Phys. Chem. Chem. Phys. 2013, 15, 5022-5029.

[26] L. M. Yang, V. Bacic, I. A. Popov, A. I. Boldyrev, T. Heine, T. Frauenheim, E. Ganz, 
J. Am. Chem. Soc. 2015, 137, 2757-2762.

[27] Y. Grin, A. Savin, B. Silvi, in The Chemical Bond (Eds.: G. Frenking, S. Shaik), Wiley-VCH, 2014, pp. 345-382.

[28] A. Filippetti, V. Fiorentini, Phys. Rev. B 2005, 72, 035128.

[29] L. Pan, B. Song, J. Sun, L. Zhang, W. Hofer, S. Du, H. J. Gao, J. Phys.: Condens. Matter 2013, 25, 505502.

\section{Table of Contents}

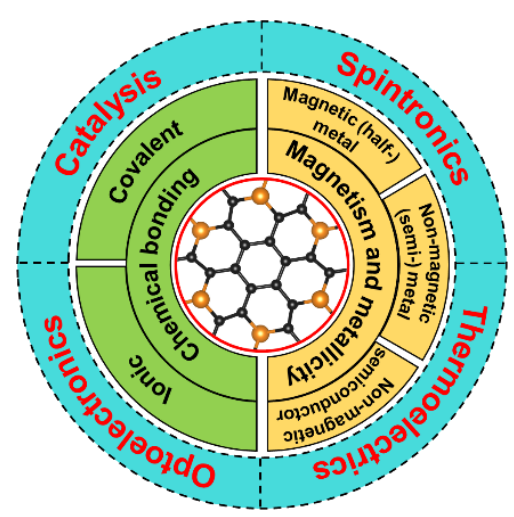

Regarded as the newest class of two-dimensional (2D) carbon-based crystals, 33 types of 2D graphitic metal carbides ( $\mathrm{g}-\mathrm{MCs}$ ) with honeycomb-in-honeycomb structures were proposed to date, which show a myriad of electronic and magnetic properties and may have applications in catalysis, spintronics, thermoelectrics, or optoelectronics. Also, the chemical bonding and origin of the intriguing magnetism of 2D g-MCs were systematically studied. 